\title{
CONSTRAINTS ON THE COMPACT OBJECT MASS IN THE ECLIPSING HIGH-MASS X-RAY BINARY XMMU J013236.7+303228 IN M 33
}

\author{
Varun B. Bhalerao ${ }^{1}$, Marten H. van KerkwiJK ${ }^{2}$, and Fiona A. Harrison ${ }^{1}$ \\ ${ }^{1}$ Cahill Center for Astrophysics, California Institute of Technology, Pasadena, CA 91125, USA \\ 2 Department of Astronomy and Astrophysics, University of Toronto, 50 St. George Street, Toronto, ON M5S 3H4, Canada \\ Received 2012 May 8; accepted 2012 June 28; published 2012 August 29
}

\begin{abstract}
We present optical spectroscopic measurements of the eclipsing high-mass X-ray binary (HMXB) XMMU J013236.7+303228 in M 33. Based on spectra taken at multiple epochs of the 1.73 day binary orbital period we determine physical as well as orbital parameters for the donor star. We find the donor to be a B1.5IV subgiant with effective temperature $T=22,000-23,000 \mathrm{~K}$. From the luminosity, temperature, and known distance to M 33 we derive a radius of $R=8.9 \pm 0.5 R_{\odot}$. From the radial-velocity measurements, we determine a velocity semi-amplitude of $K_{\mathrm{opt}}=63 \pm 12 \mathrm{~km} \mathrm{~s}^{-1}$. Using the physical properties of the B star determined from the optical spectrum, we estimate the star's mass to be $M_{\text {opt }}=11 \pm 1 M_{\odot}$. Based on the X-ray spectrum, the compact companion is likely a neutron star, although no pulsations have yet been detected. Using the spectroscopically derived B star mass we find the neutron star companion mass to be $M_{\mathrm{X}}=2.0 \pm 0.4 M_{\odot}$, consistent with the neutron star mass in the HMXB Vela X-1, but heavier than the canonical value of $1.4 M_{\odot}$ found for many millisecond pulsars. We attempt to use as an additional constraint that the B star radius inferred from temperature, flux, and distance should equate to the Roche radius, since the system accretes by Roche lobe overflow. This leads to substantially larger masses, but by trying to apply the technique to known systems, we find that the masses are consistently overestimated. Attempting to account for that in our uncertainties, we derive $M_{\mathrm{X}}=2.2_{-0.6}^{+0.8} M_{\odot}$ and $M_{\mathrm{opt}}=13 \pm 4 M_{\odot}$. We conclude that precise constraints require detailed modeling of the shape of the Roche surface.
\end{abstract}

Key words: binaries: eclipsing - stars: individual: XMMU J013236.7+303228 - X-rays: binaries

Online-only material: color figures

\section{INTRODUCTION}

The range of possible neutron star (NS) masses depends on many factors, such as the initial mass of the progenitor's stellar core, the details of the explosion (in particular mass accretion as the explosion develops), subsequent mass accretion from a binary companion, and the pressure-density relation, or equation of state (EOS), of the NS matter. On the low-mass end, producing a neutron star requires the progenitor's core to exceed the Chandrasekhar mass, which depends on the uncertain electron fraction. Theoretical models place this minimum mass in the range $M_{\text {core }} \gtrsim 0.9-1.3 M_{\odot}$ (Timmes et al. 1996). The largest possible NS mass depends on the unknown physics determining the EOS-for example, whether kaon condensates or strange matter can form in the interior (see, for example, Lattimer \& Prakash 2005). The highest-mass NS to date with an accurate measurement weighs $1.97 \pm 0.04 M_{\odot}$ (Demorest et al. 2010), which already rules out the presence of exotic hadronic matter at the nuclear saturation density (Demorest et al. 2010; Lattimer et al. 2010).

Testing the predictions of supernova models, binary evolution models, and finding objects at the extremes of the mass spectrum require determining NS masses in a variety of systems with differing progenitor masses and evolutionary history. Neutron stars accompanying either a high-mass star or another neutron star are thought to have accreted little to no matter over their lifetimes. In contrast, neutron stars in low-mass X-ray binaries and millisecond pulsars, typically in close orbits around a white dwarf, have undergone extended accretion periods that will make the current mass exceed that at birth. Different types of binaries will also have different average NS progenitor masses.
High-mass X-ray binaries (HMXBs) — binaries containing a neutron star and a massive $\left(\simeq 20 M_{\odot}\right)$ companion-are particularly interesting systems in which to pursue mass measurements. In most cases, the NS progenitor will have been more massive than the observed donor star, yielding a relatively high-mass pre-supernova core. Furthermore, the NS mass will be close to the birth mass, since even for Eddington rates $\leqslant 0.1 M_{\odot}$ can be accreted in the $\sim 10^{7} \mathrm{yr}$ lifetime of the OB companion. Indeed, among the five HMXBs with reasonably secure masses, one (Vela X-1) has $M=1.8 M_{\odot}$ (Barziv et al. 2001; Quaintrell et al. 2003), indicating that this NS may have been born heavy.

Determining NS masses in HMXBs is, however, difficult. In compact object binaries (NS-NS, NS-white-dwarf), highly precise mass measurements can be obtained from relativistic effects like the precession of periastron (Freire et al. 2008) or measurement of the Shapiro delay (Demorest et al. 2010). In HXMBs, however, accurate mass measurements are limited to eclipsing systems where orbital parameters for both the NS and its stellar companion can be measured. For the NS this is done through X-ray or radio pulse timing, and for the companion through radial-velocity measurements derived from Doppler shifts in the stellar lines. In the event pulsations are not detected, the NS mass can still be determined if good spectra are available to estimate the mass of the optical component. In rare cases where the distance to the binary is known, this provides an independent constraint on the physical scale of the system-for example, by calculating the absolute magnitude of the components. However, calculating masses from such constraints is model dependent.

In this paper, we present optical spectroscopic measurements of the donor star in the eclipsing HMXB XMMU J013236.7+ 303228 using the Low Resolution Imaging Spectrograph 


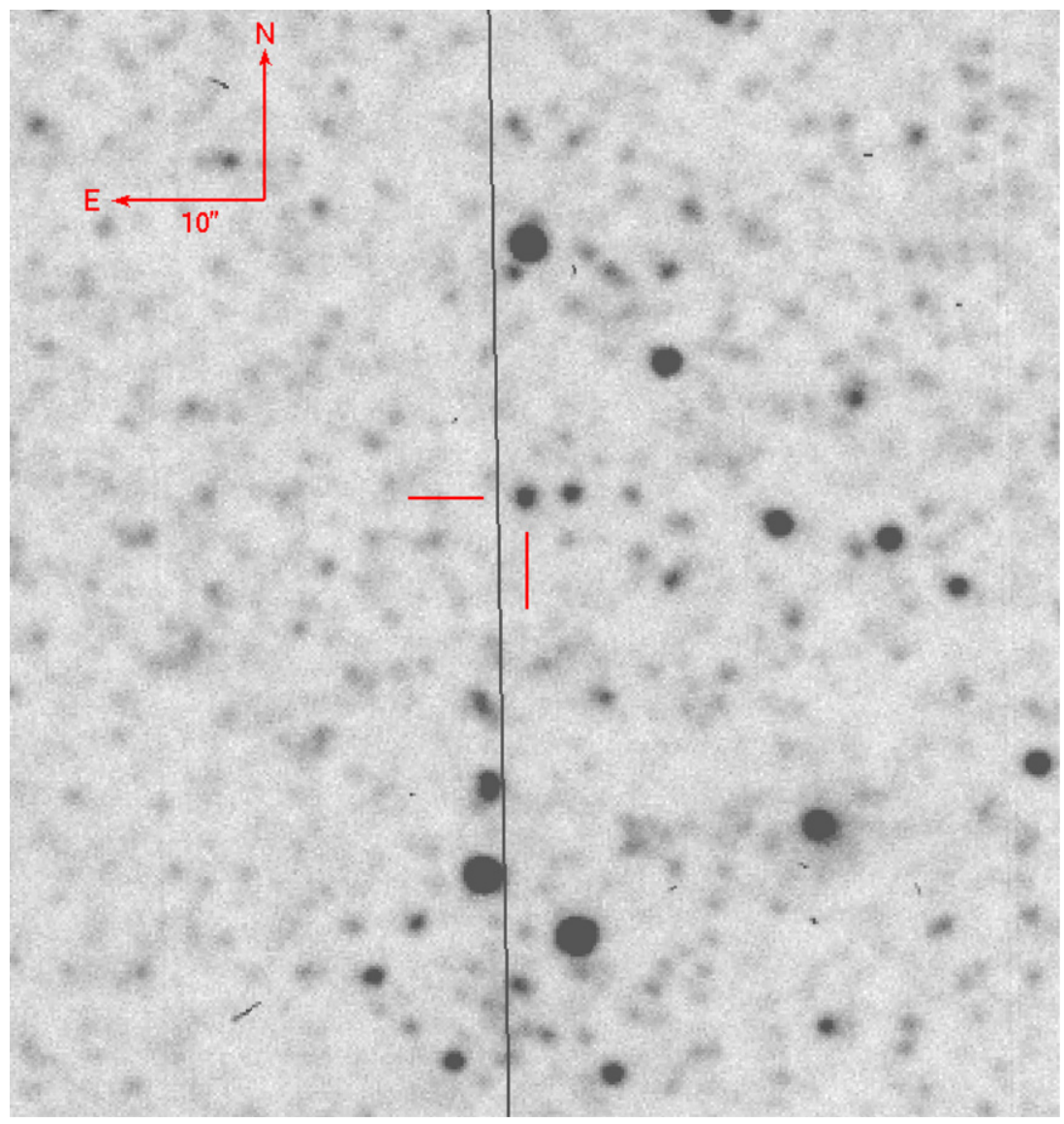

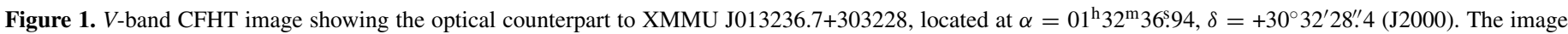
was obtained from the CFHT online data archive, and a WCS was added using astrometry.net (Lang et al. 2010).

(A color version of this figure is available in the online journal.)

(LRIS) on the $10 \mathrm{~m}$ Keck-I telescope (Oke et al. 1995) aimed at determining the mass of the compact companion. XMMU J013236.7+303228 was discovered by Pietsch et al. (2004) in their XMM-Newton survey of M 33. In follow-up observations, Pietsch et al. (2006, 2009) identified it as an eclipsing HMXB with a 1.73 day period. The X-ray spectrum is hard, and the shape implies that the compact object is a neutron star. However, no pulsations were detected in the X-ray data, so a black hole cannot be ruled out (Pietsch et al. 2009). Shporer et al. (2006) discovered an optical counterpart (Figure 1) which shows variability consistent with ellipsoidal modulation of the OB star. Given high-quality spectra we are able to obtain a spectroscopic mass for the donor, and therefore determine the compact object mass. Using the known distance to M 33 combined with the B star's luminosity and temperature we derive a physical radius, which we equate with the Roche radius based on the observation that accretion is occurring via Roche lobe overflow. This provides an additional orbital constraint that we use to independently estimate the compact object mass.

\section{OBSERVATIONS AND DATA REDUCTION}

We observed XMMU J013236.7+303228 on UT 2009 October 16 and 17, with LRIS on the $10 \mathrm{~m}$ Keck-I telescope (Oke et al. 1995), with upgraded blue (McCarthy et al. 1998; Steidel et al. 2004) and red cameras (Rockosi et al. 2010), covering a wavelength range from $3200 \AA$ to $9200 \AA$. We set up LRIS with the $600 / 4000$ grism on the blue side and the $600 / 7500$ grating on the red side to get dispersions of
$0.6 \AA$ pixel $^{-1}$ and $0.8 \AA$ pixel $^{-1}$, respectively (Table 1 ). To maximize stability of the spectra, we used the "stationary rotator mode," where the instrument rotator was held fixed near zero degrees rather than tracking the parallactic angle while observing. Atmospheric dispersion was compensated for by the atmospheric dispersion corrector (ADC). We acquired a total of 28 spectra of the target, with exposure times ranging from 300 to $1800 \mathrm{~s}$. The spectrophotometric standard EG 247 was observed for flux calibration.

The data were reduced in IRAF. ${ }^{3}$ The spectra were trimmed and bias subtracted using overscan regions. No flat fielding was applied. Cosmic rays were rejected using L.A.Cosmic (van Dokkum 2001). Atmospheric lines are stable to tens of meters per second (Figueira et al. 2010), so the wavelength solution for the red side was derived using sky lines for each image. For the blue side, the wavelength solution was derived from arcs taken at the start of the night. The spectra were then rectified and transformed to make the sky lines perpendicular to the trace to ensure proper sky subtraction. The wavelength solutions for arcs taken at various points during the night are consistent with each other to a tenth of a pixel, with only an offset between different arcs. We corrected for this offset after extracting the spectra, by using the $5577.34 \AA$ [O I] line. The spectra were extracted with APALL, and flux calibrated with data for EG 247 and the standard IRAF lookup tables. We further tweaked the flux calibration by using an EG 247 model spectrum from the

\footnotetext{
3 http://iraf.noao.edu/
} 
Table 1

Details of Individual Exposures

\begin{tabular}{|c|c|c|c|c|c|c|}
\hline Image Name & MJD & $\begin{array}{l}\text { Seeing }^{\mathrm{a}} \\
(\operatorname{arcsec})\end{array}$ & $\mathrm{S} / \mathrm{N}$ & $\begin{array}{l}\text { Heliocentric Velocity } \\
\qquad\left(\mathrm{km} \mathrm{s}^{-1}\right)\end{array}$ & $\chi^{2}$ & Notes $^{\mathrm{c}}$ \\
\hline b091016_0062 & 55120.276 & 1.0 & 5.2 & $-106 \pm 24$ & 0.96 & Rejected: logs, poor $\mathrm{S} / \mathrm{N}$ \\
\hline b091016_0064 & 55120.305 & 1.0 & 23.0 & $-32 \pm 5$ & 1.08 & Selected \\
\hline b091016_0066 & 55120.333 & 1.0 & 22.8 & $-57 \pm 5$ & 1.20 & Selected \\
\hline b091016_0080 & 55120.394 & 1.0 & 22.7 & $-101 \pm 6$ & 1.36 & Rejected: $\log s$ \\
\hline b091016_0082 & 55120.425 & 1.1 & 20.1 & $-50 \pm 6$ & 1.16 & Selected \\
\hline b091016_0087 & 55120.457 & 1.1 & 13.7 & $-47 \pm 9$ & 1.09 & Selected \\
\hline b091016_0088 & 55120.474 & 1.4 & 4.2 & $-65 \pm 30$ & 1.14 & Rejected: logs, poor $\mathrm{S} / \mathrm{N}$ \\
\hline b091016_0098 & 55120.501 & 1.2 & 18.3 & $-103 \pm 6$ & 1.08 & Rejected: $\operatorname{logs}$ \\
\hline b091016_0099 & 55120.524 & 1.1 & 19.0 & $-68 \pm 6$ & 1.02 & Selected \\
\hline b091016_0106 & 55120.560 & 1.2 & 15.5 & $-131 \pm 8$ & 1.22 & Selected \\
\hline b091016_0108 & 55120.586 & 1.1 & 11.4 & $-119 \pm 11$ & 1.00 & Selected \\
\hline b091016_0110 & 55120.608 & 1.2 & 6.3 & $-128 \pm 24$ & 1.03 & Rejected: $\operatorname{logs}$, poor $\mathrm{S} / \mathrm{N}$ \\
\hline b091017_0057 & 55121.279 & 0.9 & 11.3 & $-42 \pm 11$ & 0.99 & Rejected: logs \\
\hline b091017_0059 & 55121.305 & 0.7 & 18.8 & $-96 \pm 6$ & 0.94 & Selected \\
\hline b091017_0060 & 55121.327 & 0.9 & 19.6 & $-79 \pm 6$ & 0.93 & Selected \\
\hline b091017_0068 & 55121.367 & 0.8 & 22.5 & $-75 \pm 5$ & 0.93 & Selected \\
\hline b091017_0069 & 55121.389 & 0.8 & 22.5 & $-61 \pm 5$ & 0.97 & Selected \\
\hline b091017_0071 & 55121.419 & 1.0 & 19.5 & $-77 \pm 6$ & 0.99 & Selected \\
\hline b091017_0078 & 55121.457 & 1.0 & 19.7 & $-57 \pm 6$ & 0.95 & Selected \\
\hline b091017_0079 & 55121.478 & 1.1 & 16.9 & $-27 \pm 7$ & 1.03 & Selected \\
\hline b091017_0081 & 55121.507 & 1.1 & 18.0 & $-46 \pm 7$ & 0.98 & Selected \\
\hline b091017_0087 & 55121.541 & 1.1 & 17.2 & $-44 \pm 7$ & 1.02 & Selected \\
\hline b091017_0089 & 55121.567 & 1.1 & 15.1 & $-53 \pm 8$ & 1.03 & Selected \\
\hline b091017_0090 & 55121.589 & 1.1 & 8.7 & $-70 \pm 13$ & 0.89 & Rejected: poor S/N \\
\hline b091017_0091 & 55121.600 & 1.1 & 8.4 & $-32 \pm 15$ & 0.88 & Rejected: poor S/N \\
\hline
\end{tabular}

Notes. LRIS was set up with a $1^{\prime \prime}$ slit, the D560 dichroic and clear filters on both red and blue arms. To maximize stability, we used the stationary rotator mode. The $600 / 4000$ grism gives a dispersion of $0.61 \AA$ pixel $^{-1}$ on the blue side. On the red side, we configured the $600 / 7500$ grating at 27.70 (central wavelength $7151 \AA$ ) and get dispersion of $0.80 \AA$ pixel $^{-1}$.

a Seeing was measured as the median FWHM of Gaussians fitted to the trace of the blue-side spectrum at several points.

${ }^{\mathrm{b}}$ Errors quoted here do not include an additional $15 \mathrm{~km} \mathrm{~s}^{-1}$ error to be added in quadrature due to the motion of the star on the slit.

c Spectra were not included in the final analysis if either the observing logs mentioned that the star had moved from the slit or if the signal-to-noise ratio $(\mathrm{S} / \mathrm{N})$ per pixel in the extracted spectrum was below 10.

HST Calibration Database Archive. ${ }^{4}$ We used just one standard spectrum per night and enabled airmass correction in IRAF during flux calibration.

The final spectrum is shown in Figures 2 and 3. The signalto-noise ratio $(\mathrm{S} / \mathrm{N})$ per pixel is $>10$ for most blue-side spectra (Table 1). The first night, we experienced some tracking issues with the telescope, so the target did not remain well centered on the slit at all times. A similar problem was experienced for the first exposure on the next night, where the object was at a high airmass. In all the following discussions, we reject some such spectra based on observing logs and spectra with $\mathrm{S} / \mathrm{N}$ per pixel $<10$ (Table 1$)$.

\section{DONOR STAR PARAMETERS AND ORBIT}

We determine the best-fit stellar parameters and orbital solution using an iterative technique. First, we estimate a spectral type for the primary (donor) from individual spectra. We use appropriate spectral templates to calculate the orbital solution (Section 3.2). Next, we shift spectra to the rest frame and combine them to get a higher quality spectrum. We calculate stellar parameters from this combined spectrum and use a template spectrum with these refined parameters to recalculate

\footnotetext{
4 ftp://ftp.stsci.edu/cdbs/current_calspec/
}

the velocities. In this section, we describe the final iterations of both these steps.

\subsection{Stellar Parameters}

Based on photometry of XMMU J013236.7+303228, Pietsch et al. (2009) estimate that the companion is a $10.9 M_{\odot}$ object with $T_{\text {eff }}=33,000 \mathrm{~K}$ and $\log (g)=4.5$, with $\chi^{2}=2.4$ for their best-fit model. They then assume a distance of $795 \mathrm{kpc}$ to M 33, and calculate that the star has an absolute magnitude $M_{V} \sim-4.1$ and the line-of-sight extinction is $A_{V}=0.6$, so derive a stellar radius of $8.0 R_{\odot}$.

We deduce the spectral type by comparing our spectra to Walborn \& Fitzpatrick (1990) and the Gray spectral atlas. ${ }^{5}$ The absence of He II lines (Figure 2) implies a spectral type later than O, while the relative strengths of the $\mathrm{Mg}$ II $4482 \AA / \mathrm{He}_{\text {I }} 4471 \AA$ lines point to a spectral type earlier than B3. The strength of $\mathrm{He}$ I lines and a weak feature at $4420 \AA$ indicate a spectral type around B1 for main-sequence stars. A bump blueward of H8 $3889 \AA$ is characteristic of spectral type B2. The weakness of C III $4650 \AA$ and the relative strengths of $\mathrm{C}$ III/O II near $4650 \AA$ refine the spectral type to between B1 and B2 for both dwarfs and giants. Finally, the weakness of Si IV H $\delta 4101 \AA$ gives a spectral type

\footnotetext{
5 http://ned.ipac.caltech.edu/level5/Gray/frames.html
} 


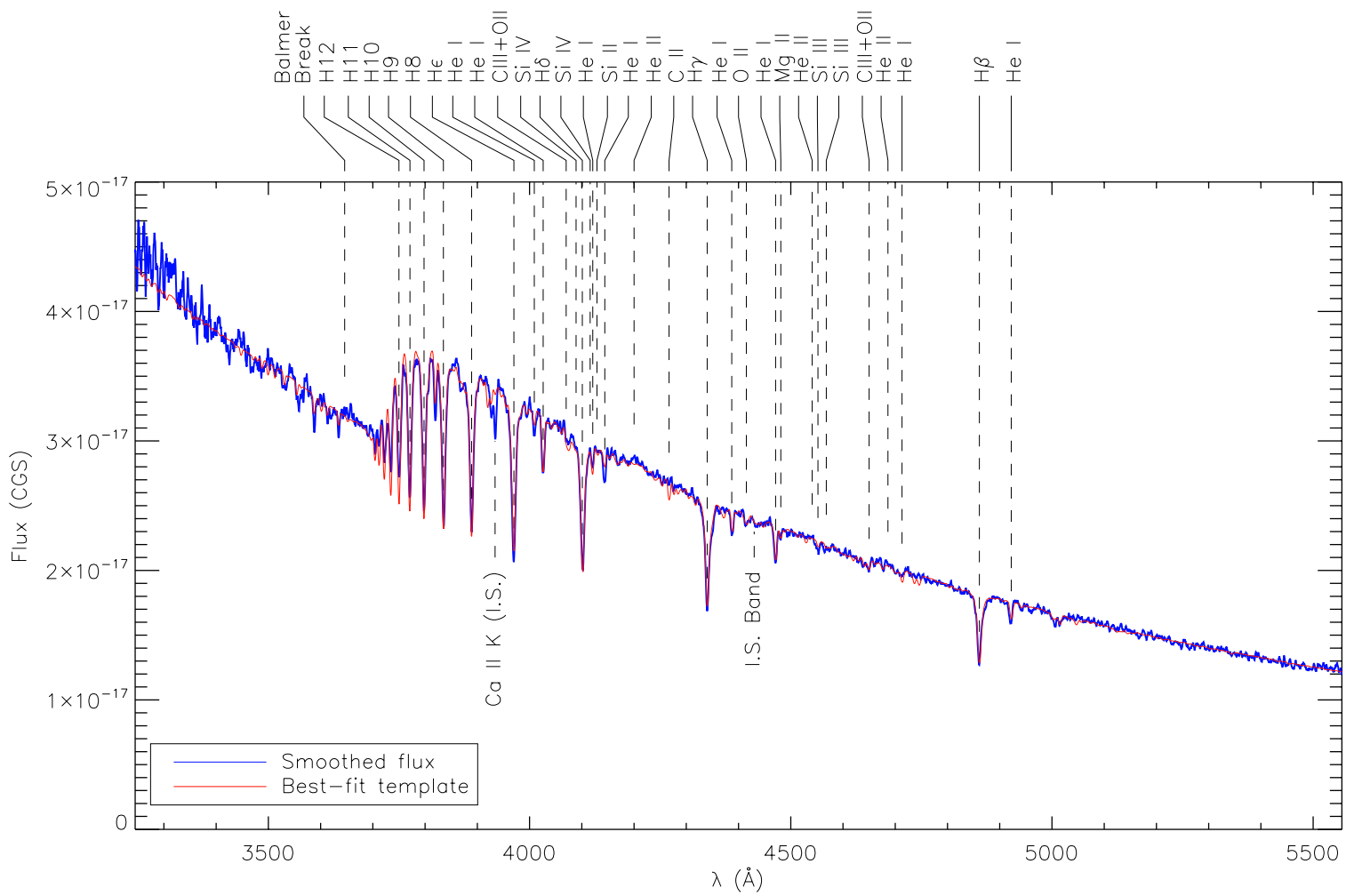

Figure 2. Observed spectrum and best-fit model for XMMU J013236.7+303228. The blue line is the average of the 10 good spectra obtained on UT 2009 October 17, shifted to the rest wavelength using velocities from Table 1 . The spectrum is smoothed with a 5 pixel ( $3 \AA$ ) boxcar for plotting. The thin red line is the best-fit template spectrum with $T=22,000 \mathrm{~K}, \log (g)=3.5, v_{\text {rot }} \sin i=250 \mathrm{~km} \mathrm{~s}^{-1}$, solar metallicity. The template is reddened using $A_{V}=0.395$ and scaled appropriately. Shifting the spectra to the rest frame blurs out the Ca II interstellar line and the $4430 \AA$ Anterstellar band. The observed higher Balmer lines are less strong than those of the model, suggesting that the surface gravity is slightly higher than $\log g=3.5$ (consistent with our estimated spectral type and with our fit results; see the text). (A color version of this figure is available in the online journal.)

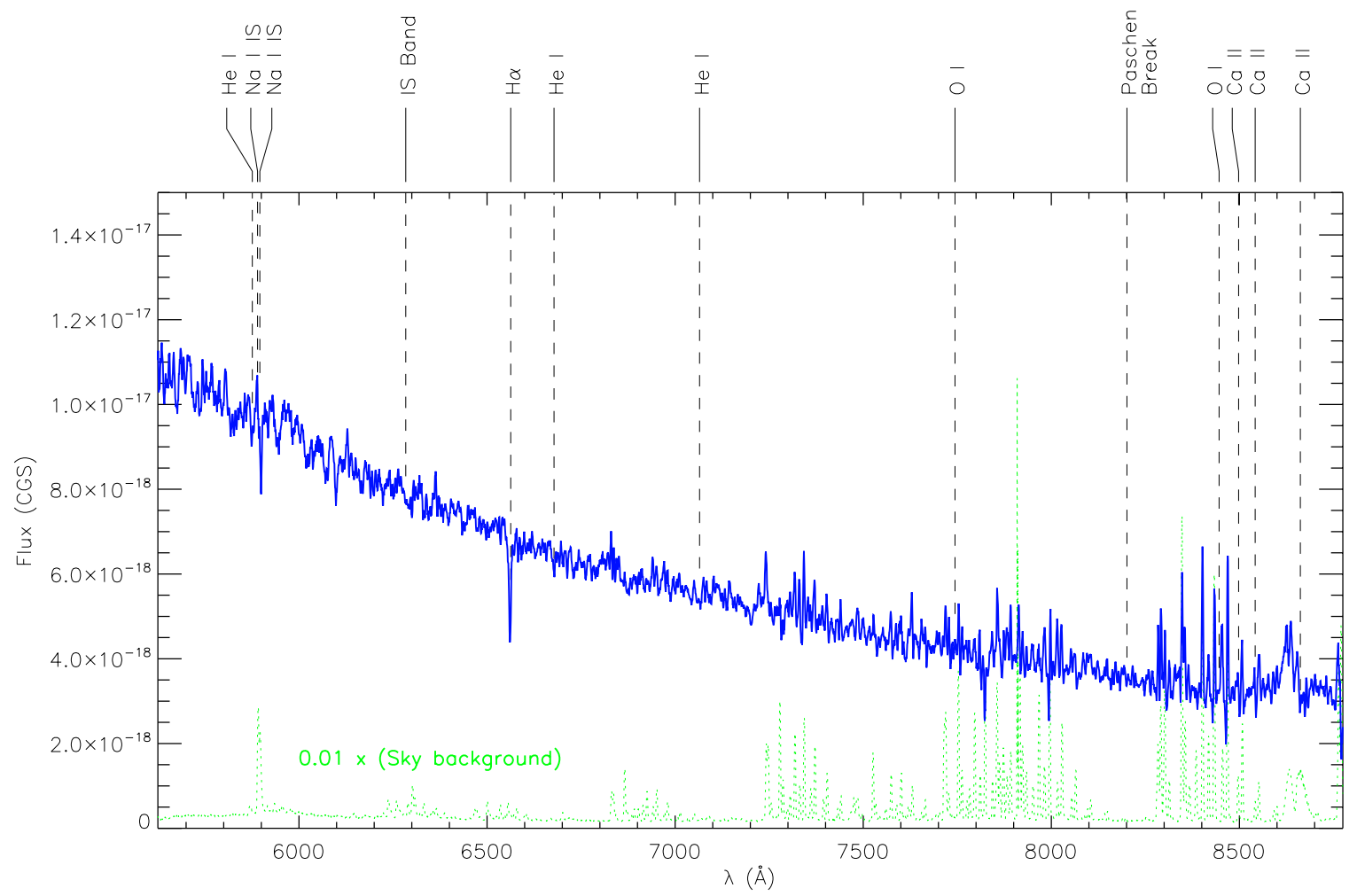

Figure 3. Observed red spectrum for XMMU J013236.7+303228. The blue line is the median combination of the 48 spectra obtained on UT 2009 October 17 , shifted to the rest wavelength using the velocity solution from Table 2 . The flux axis differs from Figure 2 . The spectrum is smoothed with a 5 pixel ( $4 \AA)$ boxcar for plotting. The source is much fainter than the sky in this wavelength region. For reference, the green dashed line shows the extracted sky spectrum, scaled down by a factor of 100 for plotting. We do not use the red-side spectra for any fitting.

(A color version of this figure is available in the online journal.) 
of B1.5. To determine the luminosity class, we note that the O II 4415-4417 $\AA$ and Si III $4552 \AA$ lines are present, but are weak as compared to $\mathrm{He}$ I 4387 . We conclude that the donor is a B1.5IV star, with rough uncertainties of 0.5 spectral subclasses and one luminosity class.

Tabulated values of stellar parameters are usually provided for luminosity class III and V stars. For B1.5V stars, $T \approx$ $23,000 \mathrm{~K}, \log (g)=4.14$, and $M_{V}=-2.8$. For B1.5III stars, $T \approx 22,000 \mathrm{~K}, \log (g)=3.63$, and $M_{V}=-3.4(\operatorname{Cox} 2000)$. The B1.5IV target will have values intermediate to these. This inferred temperature is significantly cooler than $T \sim 33,000 \mathrm{~K}$ reported by Pietsch et al. (2009). However, the absence of He II lines at, e.g., 4541 and $4686 \AA$ clearly excludes such a high temperature.

The colors of a star depend on its temperature and surface gravity. These expected colors can be compared to the observed colors to calculate the reddening and extinction. The expected color is $(B-V)_{0}=-0.224$ for a $22,000 \mathrm{~K}$ subgiant star, and $(B-V)_{0}=-0.231$ for a $23,000 \mathrm{~K}$ main-sequence star (Bessell et al. 1998). From Pietsch et al. (2009), the mean magnitudes are $m_{g^{\prime}}=21.03 \pm 0.02$ and $m_{r^{\prime}}=21.36 \pm 0.02$. Using the Jester et al. (2005) photometric transformations for blue, $U-B<0$ stars, ${ }^{6} m_{V}=21.21 \pm 0.03$ and $(B-V)_{\text {obs }}=-0.09 \pm 0.04$. The color excess is $E(B-V)=(B-V)_{\mathrm{obs}}-(B-V)_{0}=0.14 \pm$ 0.04 . Using the standard ratio of total-to-selective extinction, $R_{V}=3.1$, we get $A_{V}=0.43 \pm 0.12$. For comparison, the foreground extinction to M 33 is $A_{V}=0.22$.

We measure various stellar parameters by fitting our combined, flux-calibrated spectrum with model atmospheres (taking into account the instrumental broadening; see Section 3.2). For a Roche-lobe-filling companion (discussed in Section 4), we expect a radius of $6-10 R_{\odot}$, surface gravity $\log (g) \simeq 3.7$ (consistent with our luminosity class), and projected rotational velocity $v_{\text {rot }} \sin i \simeq 250 \mathrm{~km} \mathrm{~s}^{-1}$. We use these values as starting points to select model atmospheres from a grid calculated by Munari et al. (2005). These templates are calculated in steps of 0.5 dex in $\log (g)$, so we use models with $\log (g)=3.5,4.0$. For the initial fit, we assume $v_{\text {rot }} \sin i=250 \mathrm{~km} \mathrm{~s}^{-1}$ and solar metallicity. The only free parameters are a normalization and an extinction. We use extinction coefficients from Cox (2000), assuming $R_{V}=3.1$. We find that the best-fit model for $\log (g)=3.5$ has $T=22,100 \pm 40 \mathrm{~K}$ and $A_{V}=0.401(3)$, with $\chi^{2} /$ dof $=1.13$ for 3600 degrees of freedom, while for $\log (g)=4.0$, we get $T=23,500 \pm 50 \mathrm{~K}$ and $A_{V}=0.425(3)$ with $\chi^{2} /$ dof $=1.22$. The temperatures are consistent with those expected for a B1.5 subgiant star, and the extinction is within the range derived from photometric measurements. Pietsch et al. (2009) obtained a higher extinction for the target, which explains why they estimated the source temperature to be higher. Munari et al.'s (2005) templates are calculated in temperature steps of $1000 \mathrm{~K}$ in this range. For further analysis, we use the best-fit template: $\log (g)=3.5$ and $T=22,000 \mathrm{~K}$. Since $\log (g)$ is slightly higher than this value, for completeness we also give results using the best-fit template for $\log (g)=4.0$, which has $T=23,000 \mathrm{~K}$. For both these templates, the best-fit extinction is $A_{V}=0.395(3)$. We then keep $T$ and $\log (g)$ constant and vary $v_{\text {rot }} \sin i$. For both the $\log (g)$ and $T$ combinations, we measure $v_{\text {rot }} \sin i=260 \pm 5 \mathrm{~km} \mathrm{~s}^{-1}$. Finally, using the same templates but with varying metallicity, we get the best fits for $[\mathrm{M} / \mathrm{H}]=$ 0 . The 0.5 dex steps in $[\mathrm{M} / \mathrm{H}]$ are too large to formally fit for uncertainties.

$\overline{6 V=g-0.59(g-r)-0.01 \pm 0.01 ; B-V}=0.90(g-r)+0.21 \pm 0.03$.
Next, we calculate the luminosity of the object to obtain a radius-temperature relation. The distance modulus to $M 33$ is $(m-M)_{\mathrm{M} 33}=24.54 \pm 0.06(d=809 \mathrm{kpc} ;$ McConnachie et al. 2005; Freedman et al. 2001), which gives, accounting for the reddening of $A_{V}=0.4, M_{V}=-3.74 \pm 0.07$. The bolometric luminosity of a star is related to its temperature and radius by $L_{\text {bol }} \propto R^{2} T^{4}$. To obtain the visual luminosity, one must apply a temperature-dependent bolometric correction, $\mathrm{BC}=M_{\mathrm{bol}}-$ $M_{V}$. Torres (2010) gives formulas for bolometric correction as a power series in $\log (T)$. We calculate $\mathrm{BC}=-2.11(-2.21)$ for $T=22,000(23,000) \mathrm{K}$ (which are consistent with Bessell et al.'s (1998) tables for main-sequence stars). After some basic algebra, we obtain

$$
\begin{aligned}
5 \log & \left(\frac{R}{R_{\odot}}\right)+10 \log \left(\frac{T}{T_{\odot}}\right)+\mathrm{BC}(T) \\
& =M_{\mathrm{bol}, \odot}-m_{V}+(m-M)_{\mathrm{M} 33}+A_{V} \\
& =8.48 \pm 0.07 .
\end{aligned}
$$

Here, $M_{\mathrm{bol}, \odot}=4.75$ (Bessell et al. 1998). The resultant radius-temperature relationship is shown in Figure 4. For $T=22,000(23,000) \mathrm{K}$, we infer $R=9.1(8.7) \pm 0.3 R_{\odot}$. The absolute magnitude and radius are both consistent with a B1.5 subgiant.

\subsection{Orbital Parameters}

We measure the radial velocities of the B star using model stellar spectra by Munari et al. (2005) as follows. For each observed spectrum, we measure the seeing using the width of the spectral trace. We then generate an instrument response function by taking a Gaussian matched to the seeing, truncating it at the slit size, and convolving it with the pixel size. Fluxcalibrated synthetic spectra (templates) are convolved with this instrument response and then redshifted to a test velocity. Then we redden the template using the measured value of extinction, $A_{V}=0.395$, with coefficients from Cox (2000). We use $\mathrm{IDL}^{7} \mathrm{mpfit}$ (Markwardt 2009) to calculate the reddening and normalization to match this spectrum with the observed spectrum and measure the $\chi^{2}$. By minimizing the $\chi^{2}$ over test velocities, we find the best-fit velocity and the error bars. This velocity is converted to a barycentric radial velocity using the baryvel routine in Astrolib (Landsman 1993). Table 1 lists the radial velocities for all spectra, measured using the best-fit stellar template: $T=22,000 \mathrm{~K}, \log g=3.5,[\mathrm{M} / \mathrm{H}]=0.0$, and $v_{\text {rot }} \sin i=250 \mathrm{~km} \mathrm{~s}^{-1}$.

Red-side spectra are not useful for radial-velocity measurement for several reasons. The flux of the B star at redder wavelengths is lower than at blue wavelengths, and there are fewer spectral lines in this range. Also, the background noise is higher, from the large number of cosmic rays detected by the LRIS red side and from intrinsic sky emission. Hence, all further discussion omits red-side spectra.

We calculate an orbital solution for the B star using these radial velocities. Owing to the short 1.73 day period of the system, we assume that the orbit must be circularized. The orbital solution is then given by

$$
v(t)=\gamma_{\mathrm{opt}}+K_{\mathrm{opt}} \sin \left(2 \pi \frac{t-T_{0}}{P}\right),
$$

where $\gamma_{\mathrm{opt}}$ is the systemic velocity, $K_{\mathrm{opt}}$ is the projected semi-amplitude of radial velocity, and $T_{0}$ is the epoch of

\footnotetext{
http://www.ittvis.com/ProductServices/IDL.aspx
} 


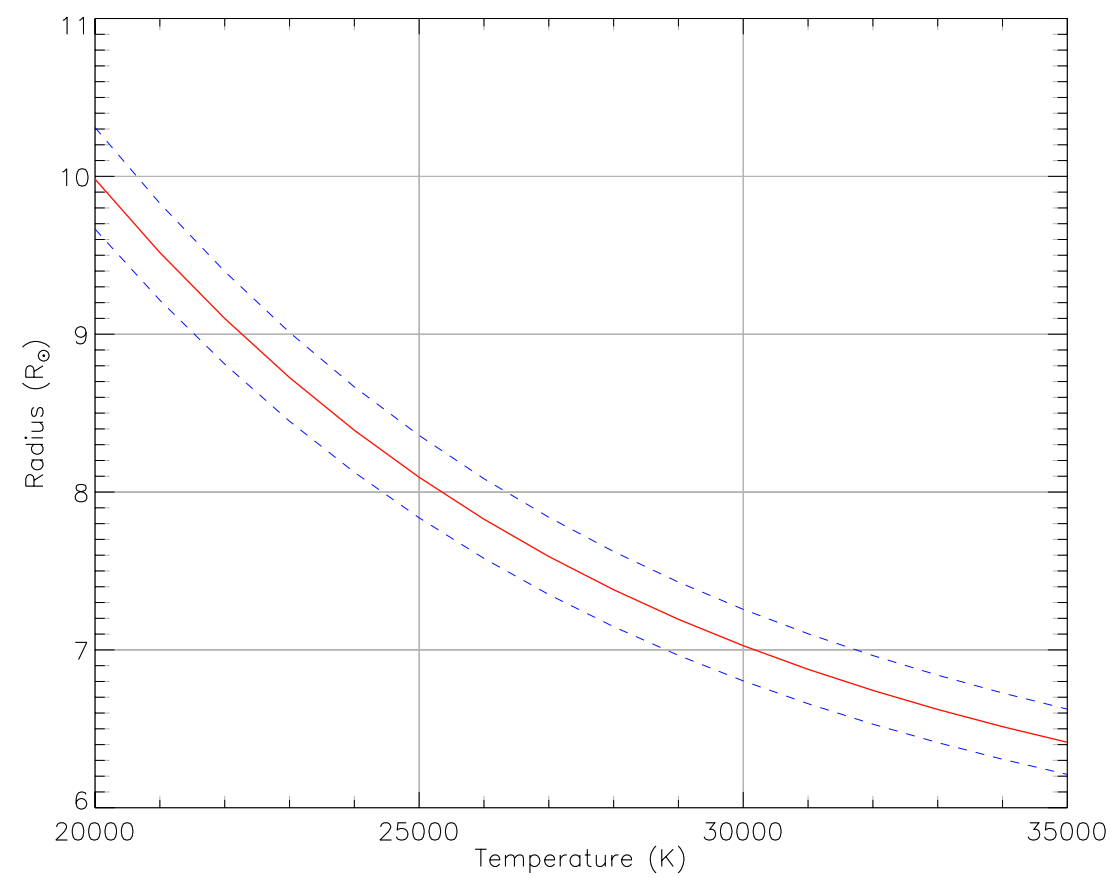

Figure 4. Radius-temperature constraint from the observed luminosity. We calculate the absolute visual magnitude from the apparent magnitude, distance modulus, and best-fit extinction. We then apply a temperature-dependent bolometric correction to calculate the bolometric magnitude. For any given temperature in this range, the uncertainty in radius is about $3 \%$.

(A color version of this figure is available in the online journal.)

mid-eclipse. We adopt $P=1.732479 \pm 0.000027$ and $T_{0}=$ $2453997.476 \pm 0.006$ from Pietsch et al. (2009). We obtain $\gamma_{\mathrm{opt}}=-80 \pm 5 \mathrm{~km} \mathrm{~s}^{-1}$ and $K_{\mathrm{opt}}=64 \pm 12 \mathrm{~km} \mathrm{~s}^{-1}$. The best fit has $\chi^{2} /$ dof $=5.1$ for 16 degrees of freedom, which is rather poor. We find that an additional error term $\Delta v=15 \mathrm{~km} \mathrm{~s}^{-1}$ needs to be added in quadrature to our error estimates to obtain $\chi_{\text {red }}^{2}=1$. We attribute this to the movement of the star on the slit. If the target has a systematic offset of 0.1 from the slit center over the entire 30 minute exposure, it shifts the line centroids by about 0.45 pixels or $18 \mathrm{~km} \mathrm{~s}^{-1}$. For comparison, van Kerkwijk et al. (2011) find a similar scatter $\left(13 \mathrm{~km} \mathrm{~s}^{-1}\right)$ in their observations of a reference star when using LRIS with a similar configuration (600/4000 grating, 0'7 slit). Future observations should orient the spectrograph slit to obtain a reference star spectrum to correct for such an offset.

When we fit Equation (2) to data including the $15 \mathrm{~km} \mathrm{~s}^{-1}$ error in quadrature, we obtain $\gamma_{\mathrm{opt}}=-80 \pm 5 \mathrm{~km} \mathrm{~s}^{-1}$ and $K_{\mathrm{opt}}=63 \pm 12 \mathrm{~km} \mathrm{~s}^{-1}$ (Figure 5, Table 2). For the epoch of observations, the uncertainty in the phase is 0.019 days. If we allow $T_{0}$ to vary, we get $T_{0, \text { fit }}=2453997.489 \pm 0.019$ (Heliocentric Julian Date), $\gamma_{\mathrm{opt}}=-80 \pm 5 \mathrm{~km} \mathrm{~s}^{-1}$, and $K_{\mathrm{opt}}=$ $64 \pm 12 \mathrm{~km} \mathrm{~s}^{-1}$. These values are consistent with those obtained using the Pietsch et al. (2009) ephemeris. Hence, for the rest of this paper, we simply assume their best-fit value for $T_{0}$.

To investigate the sensitivity of the result to the choice of the stellar template, we repeat the measurement with a variety of templates. We vary the temperature from $\mathrm{O} 9(33,000 \mathrm{~K})$ to $\mathrm{B} 3$ $(18,000 \mathrm{~K})$ spectral classes. As before, we use templates with $\log (g)=3.5,4.0, v_{\mathrm{rot}} \sin i=250 \mathrm{~km} \mathrm{~s}^{-1}$ and solar metallicity. Repeating the orbit calculations for each of these models, we find that the systemic velocity $\gamma_{\text {opt }}$ may change between the models being fitted: the extreme values are $-77 \pm 6 \mathrm{~km} \mathrm{~s}^{-1}$ and $-90 \pm 5 \mathrm{~km} \mathrm{~s}^{-1}$, a $1.7 \sigma$ difference. We suspect that the reason for this variation is the difference in shape of the continuum, as
Table 2

System Parameters for XMMU J013236.7+303228

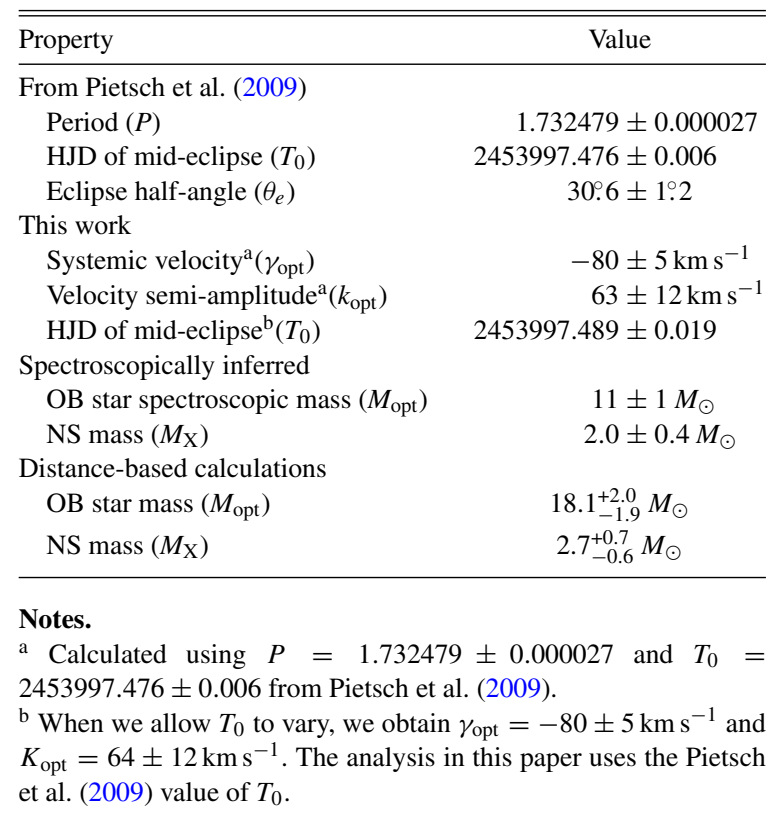

the extinction was held constant in these fits. For hotter templates with a steeper continuum, the red side of a line has lower flux than the blue side, so lowest $\chi^{2}$ will be obtained at a slightly higher redshift, as seen. The magnitude of this effect should be independent of the intrinsic Doppler shift of the spectrum and should not affect the velocity semi-amplitude $K_{\text {opt }}$. This is indeed the case: $K_{\mathrm{opt}}$ is constant irrespective of templates. The extreme values are $62 \pm 12 \mathrm{~km} \mathrm{~s}^{-1}$ and $63 \pm 12 \mathrm{~km} \mathrm{~s}^{-1}$, differing by less than $0.1 \sigma$. Dynamical calculations depend only on $K_{\mathrm{opt}}$, and hence are robust to the selection of template. 


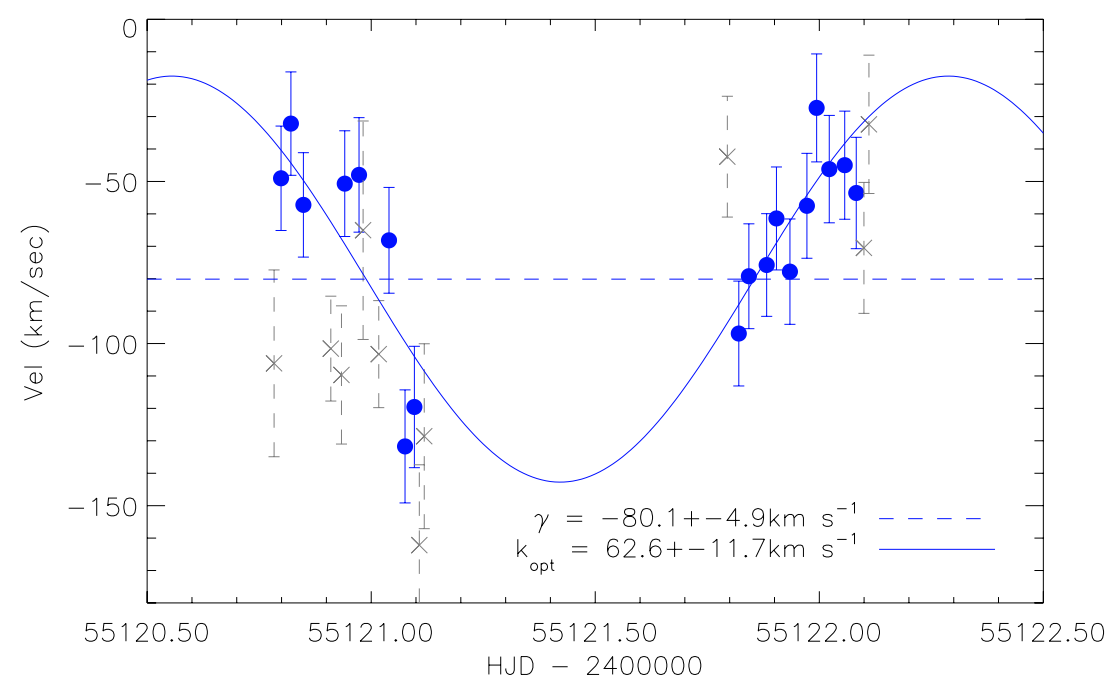

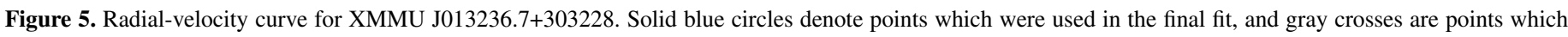

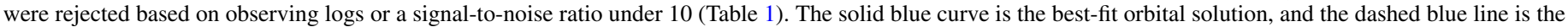
mean systemic velocity.

(A color version of this figure is available in the online journal.)

\section{COMPONENT MASSES}

The general method for accurately determining masses in X-ray binaries (Joss \& Rappaport 1984) requires measuring the orbit for both components, as well as having a constraint on the orbit inclination. In general, the mass $\left(M_{1}\right)$ of a component is expressed in terms of five parameters: the orbital period $P$, the radial-velocity semi-amplitude of the companion $\left(K_{2}\right)$, the eccentricity $e$, the orbital inclination $i$, and mass ratio $q=M_{1} / M_{2}$. The first three parameters can be readily obtained by characterizing the orbit of either component through pulse timing of the NS in the X-ray or radio, or by spectroscopically measuring the radial velocity of the optical companion at optical or infrared wavelengths. Determining the mass ratio requires measuring orbital parameters for both components: $q=K_{2} / K_{1}$. In an eclipsing system, the inclination can be constrained to be nearly edge-on $\left(i \approx 90^{\circ}\right)$, with a lower limit derived from eclipse duration and Roche lobe arguments. Using these measurements, masses of both components in the system can be directly determined without model assumptions (see, for example, van der Meer et al. 2007; Mason et al. 2011).

Because no pulsations have been detected from the compact object in XMMU J013236.7+303228 (Pietsch et al. 2009), we need one more constraint in addition to the radial-velocity semiamplitude of the donor. In Section 4.1, we use the spectroscopically inferred mass of the primary to calculate the mass of the secondary from the mass ratio. Because we know the distance to M 33, and hence to XMMU J013236.7+303228, we can also estimate the physical size of the secondary from the distance, its luminosity, and temperature. This provides a cross-check on the mass determination derived from the spectroscopic donor mass (Section 4.2).

\subsection{The Spectroscopic Method}

In the following, we will denote the masses of the compact object and donor star by $M_{\mathrm{X}}$ and $M_{\mathrm{opt}}$, respectively. The mass of the compact object $\left(M_{\mathrm{X}}\right)$ is related to the radial velocity of the $\mathrm{B} \operatorname{star}\left(K_{\mathrm{opt}}\right)$ as follows:

$$
M_{\mathrm{X}}=\frac{K_{\mathrm{opt}}^{3} P\left(1-e^{2}\right)^{3 / 2}}{2 \pi G \sin ^{3} i}\left(1+\frac{1}{q}\right)^{2},
$$

where $q=M_{\mathrm{X}} / M_{\mathrm{opt}}$ is the ratio of masses, defined so that higher values of $M_{\mathrm{X}}$ relate to higher values of $q . P$ is the orbital period of the binary, $e$ is the eccentricity, and $i$ is the inclination of the orbit. For eclipsing systems, the inclination is constrained by

$$
\sin i=\frac{\sqrt{1-\beta^{2}\left(R_{\mathrm{L}} / a\right)^{2}}}{\cos \theta_{\mathrm{e}}},
$$

where $R_{\mathrm{L}}$ is the volume radius of the Roche lobe, $a$ is the semi-major axis, and $\beta$ is the Roche lobe filling factor (Joss \& Rappaport 1984). For XMMU J013236.7+303228, the eclipse half-angle is $\theta_{\mathrm{e}}=30.6 \pm 1.2$ (Pietsch et al. 2009). Owing to the short orbital period, we assume that the orbit is circular and the B star rotation is completely synchronized with its orbit. For corotating stars, Eggleton (1983) expresses $R_{\mathrm{L}} / a$ in terms of $q$ :

$$
\frac{R_{\mathrm{L}}}{a}=\frac{0.49 q^{-2 / 3}}{0.6 q^{-2 / 3}+\ln \left(1+q^{-1 / 3}\right)} .
$$

The constant, relatively high X-ray luminosity, sustained over the non-eclipsed parts of the orbit, strongly indicates that accretion is occurring via Roche lobe overflow. In Roche lobe overflow, matter flowing through the Lagrangian point may form a disk around the compact object before being accreted onto it. This disk can occult the compact object, causing periods of low X-ray luminosity. Both these characteristics are seen in the X-ray light curves of XMMU J013236.7+303228 (Pietsch et al. 2009). If mass is being accreted onto an object in a spherically symmetric manner, the accretion rate is limited by the Eddington rate, $\dot{M}_{\text {Edd }}$, and the peak luminosity is $L_{\text {Edd }} / L_{\odot}=$ $3 \times 10^{4} M / M_{\odot}$. For a $1.5-2.5 M_{\odot}$ compact object, $L_{\text {Edd }}=$ $1.8-3 \times 10^{38} \mathrm{erg} \mathrm{s}^{-1}$. At its brightest, the source luminosity in the $0.2-4.5 \mathrm{keV}$ band was $2.0 \times 10^{37} \mathrm{erg} \mathrm{s}^{-1}$-about $0.1 L_{\text {Edd }}$. This luminosity was sustained throughout Chandra ObsID 6387, which covered about 0.72 days of the non-eclipsed orbit (Pietsch et al. 2009). Comparable flux was observed in the non-eclipsed parts of the orbit (0.73 days) in Chandra ObsID 6385. Such high luminosity sustained over significant parts of the orbit is not observed in wind-fed systems, which have typical luminosities an order of magnitude smaller. Further, the short 1.73 day orbital period is not consistent with Be X-ray binary or wind-fed 


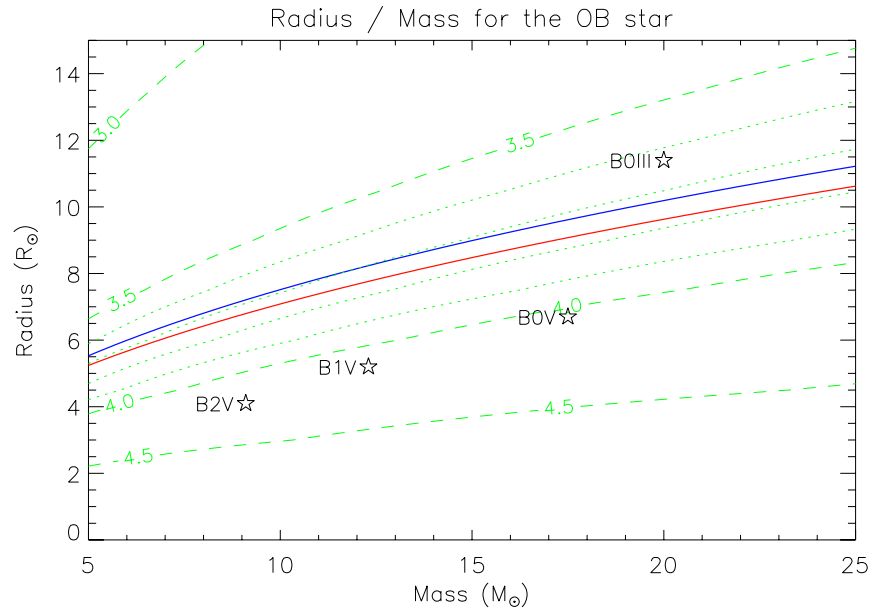

Figure 6. Mass-radius relation for the $\mathrm{OB}$ star. The solid lines show the Roche lobe radius for the OB star, assuming a $2.4 M_{\odot}$ (upper blue line) and a $1.4 M_{\odot}$ (lower red line) neutron star. The dashed green lines are contours for $\log (g)$ in steps of 0.5 dex for which synthetic spectra are available (Munari et al. 2005). In the range $3.5<\log (g)<4.0$, dotted $\log (g)$ contours are separated by steps of 0.1 dex. The stars denote the masses and radii of typical isolated B stars.

(A color version of this figure is available in the online journal.)

systems. We conclude therefore that the B star fills its Roche lobe.

The Roche lobe radius as a function of B star mass is plotted in Figure 6 for various neutron star masses. For a Roche-filling companion, we infer $3.6 \leqslant \log (g) \leqslant 3.8$. For an NS mass in the range 1.4-2.4 $M_{\odot}$ and $\mathrm{B}$ star mass $8-20 M_{\odot}$, the $\mathrm{B}$ star radius lies in the range $6-10 R_{\odot}$. Further, the assumed synchronous rotation requires the surface rotational velocity to be in the range $200 \mathrm{~km} \mathrm{~s}^{-1} \lesssim v_{\text {rot }} \lesssim 285 \mathrm{~km} \mathrm{~s}^{-1}$. These values are consistent with those derived in Section 3.1.

Figure 7 plots $M_{\mathrm{X}}$ as a function of $M_{\mathrm{opt}}$, calculated by solving Equations (3)-(5) under known constraints. If we know $M_{\mathrm{opt}}$, we can calculate $M_{\mathrm{X}}$. We use the physical properties of the primary (Section 3.1) to estimate the mass of the primary by comparing it with stellar evolutionary models, assuming that binary evolution has not drastically changed the mass-luminosity relation. First, we place the primary on an H-R diagram (Figure 8) using models by Brott et al. (2011). We conservatively allow for a $0.5 \mathrm{mag}$ error in luminosity. We also plot evolutionary tracks on a $\log (g)-T$ figure to utilize the stricter constraints on $\log (g)$ from Roche lobe arguments. From these plots, we see that the primary is approximately a $15 \mathrm{Myr}$ old, $\sim 11 M_{\odot}$ star. This is consistent with the typical mass of a B1.5IV star (Cox 2000).

For $M_{\text {opt }}=11 M_{\odot}$, we calculate $M_{\mathrm{X}}=2.0 \pm 0.4 M_{\odot}$. From evolutionary tracks, we estimate that the uncertainty in $M_{\mathrm{opt}}$ is $\sim 1 M_{\odot}$ (Figure 8), corresponding to $\Delta M_{\mathrm{X}}=0.12$, much smaller than the uncertainty arising from $\Delta K_{\mathrm{opt}}$. Adding this in quadrature with the uncertainty in the $M_{\mathrm{X}}-M_{\mathrm{opt}}$ conversion, we conclude $M_{\mathrm{X}}=2.0 \pm 0.4 M_{\odot}$.

\subsection{Masses from Roche Lobe Constraints}

In Section 3.1, we calculated the radius of the primary from its apparent magnitude, temperature, and the distance to M 33 (Equation (1)). Since the primary is filling its Roche lobe, the stellar radius is equal to the Roche lobe radius $\left(R_{\mathrm{L}}\right)$. This additional constraint can be used in Equations (3)-(5) to solve for $M_{\mathrm{X}}$ and $M_{\mathrm{opt}}$.

We calculate the probability density function (PDF) of component masses as follows. For every pair of assumed masses

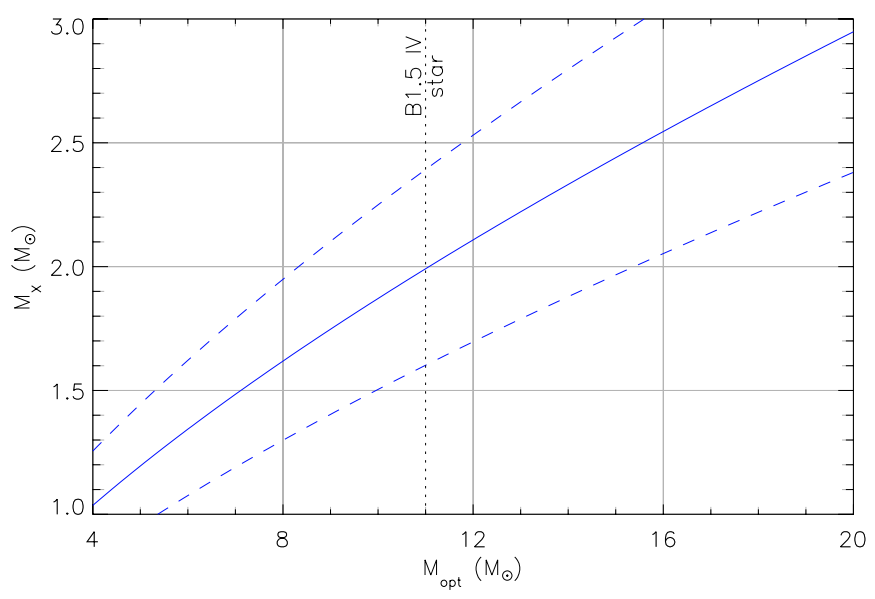

Figure 7. Solid blue line shows the compact object mass $\left(M_{\mathrm{X}}\right)$ as a function of OB star mass $\left(M_{\mathrm{opt}}\right)$. Dashed blue lines are $\pm 1 \sigma$ errors. The estimated mass of the B1.5IV primary is $11 M_{\odot}$, marked by the vertical dotted line. The corresponding mass of the neutron star is $2.0 \pm 0.4 M_{\odot}$.

(A color version of this figure is available in the online journal.)

$\left(M_{\mathrm{X}}, M_{\mathrm{opt}}\right)$, we use the period $P$ to calculate the semi-major axis $a$. Then we calculate $R_{\mathrm{L}} / a$ from Equation (5) and substitute it in Equation (4) to calculate $\sin i$. Using $P, a$, and $\sin i$ we calculate the expected semi-amplitude of the radial velocity:

$$
K_{2}=\frac{2 \pi a \sin i}{P} \frac{M_{\mathrm{X}}}{\left(M_{\mathrm{X}}+M_{\mathrm{opt}}\right)} .
$$

Next, we calculate the probability for obtaining a certain value of $R_{\mathrm{L}}$ and $K_{2}$, given the measured radius $R$ (Section 3.1) and $K_{\mathrm{opt}}$ (Section 3.2):

$$
P\left(R_{\mathrm{L}}, K_{2}\right)=\exp \left(-\frac{\left(R_{\mathrm{L}}-R\right)^{2}}{2 \cdot \Delta R^{2}}\right) \exp \left(-\frac{\left(K_{2}-K_{\mathrm{opt}}\right)^{2}}{2 \cdot \Delta K_{\mathrm{opt}}^{2}}\right) .
$$

Here, we are making a simplifying assumption that the Roche volume radius (Equation (5)) is same as the effective radius from photometry (Equation (1)). We convert this PDF to a probability density as a function of $M_{\mathrm{X}}, M_{\mathrm{opt}}$ by multiplying by the Jacobian $\partial\left(R, K_{\text {opt }}\right) / \partial\left(M_{\mathrm{X}}, M_{\text {opt }}\right)$.

The results are shown in Figure 9. Red contours show the $68.3 \%$ and $95.4 \%$ confidence intervals for masses for the bestfit template $(\log (g)=3.5$ and $T=22,000 \mathrm{~K})$. The panel on the left shows the PDF for $M_{\mathrm{X}}$ marginalized over $M_{\mathrm{opt}}$. Similarly, the lower panel shows the PDF for $M_{\mathrm{opt}}$ marginalized over $M_{\mathrm{X}}$. In these panels, the solid, dashed, and dotted lines show the peak and $68.3 \%$ and $95.4 \%$ confidence intervals, respectively. We obtain $M_{\mathrm{X}}=2.7_{-0.6}^{+0.7} M_{\odot}$ and $M_{\text {opt }}=18.1_{-1.9}^{+2.0} M_{\odot}$. For completeness, fits for the less likely scenario with $\log (g)=4.0$ and $T=23,000 \mathrm{~K}$ are shown in blue. In this case, $M_{\mathrm{X}}=$ $2.5 \pm 0.6 M_{\odot}$ and $M_{\mathrm{opt}}=16.1_{-1.7}^{+1.8} M_{\odot}$.

To test the validity of this technique, we apply it to two wellstudied targets: LMC X-4 and SMC X-1. We find that the basic application of our method is overestimating the mass (Table 3). Part of this discrepancy is likely related to equating the Roche volume radius to the effective photometric radius (Equation (7)). The Roche volume radius (Equation (5)) is the radius of a sphere with the same volume as the Roche lobe of the star. The effective photometric radius (Equation (1)) is the radius of a sphere with the same surface area as the star. Since an ellipsoid has a larger surface area than a sphere of the same volume, the actual Roche 

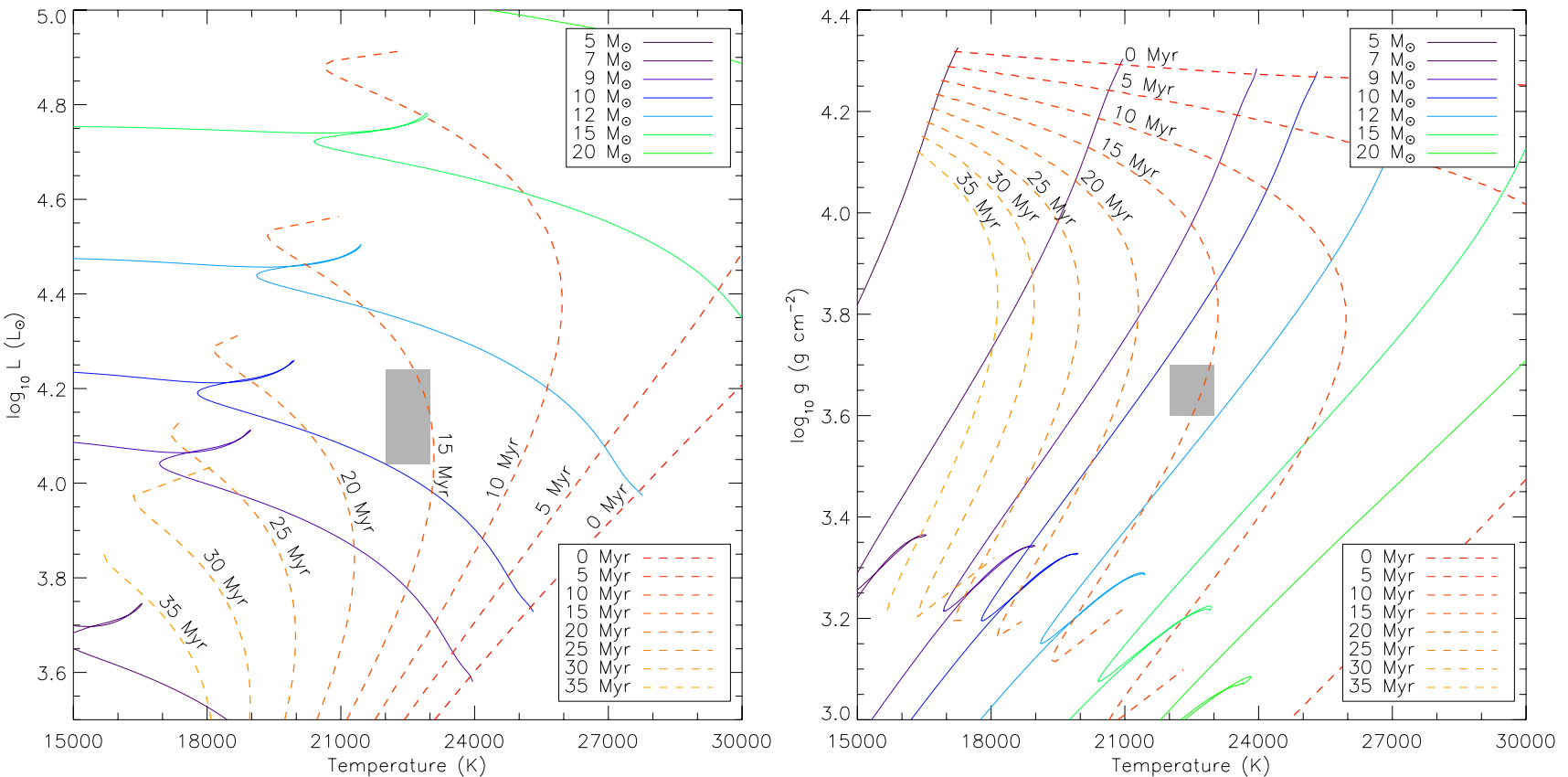

Figure 8. Evolutionary tracks and isochrones for massive stars (adapted from Brott et al. 2011). Left panel: the conventional H-R diagram with luminosity and temperature. The primary star has $22,000 \mathrm{~K} \lesssim T \lesssim 23,000 \mathrm{~K}$. We calculate luminosity from the observed $m_{V}$ and allow a 0.5 mag offset to calculate the lower limit (see the discussion in Section 4.1). This region is shown by a shaded gray box. Right panel: same as the left panel, but plotted as $\log (g)$ vs. $T$. $\log (g)$ for the primary is constrained from Roche lobe arguments (Section 3.1). From both panels, we see that the primary is consistent with a $11 M_{\odot}, 15 \mathrm{Myr}$ object.

(A color version of this figure is available in the online journal.)

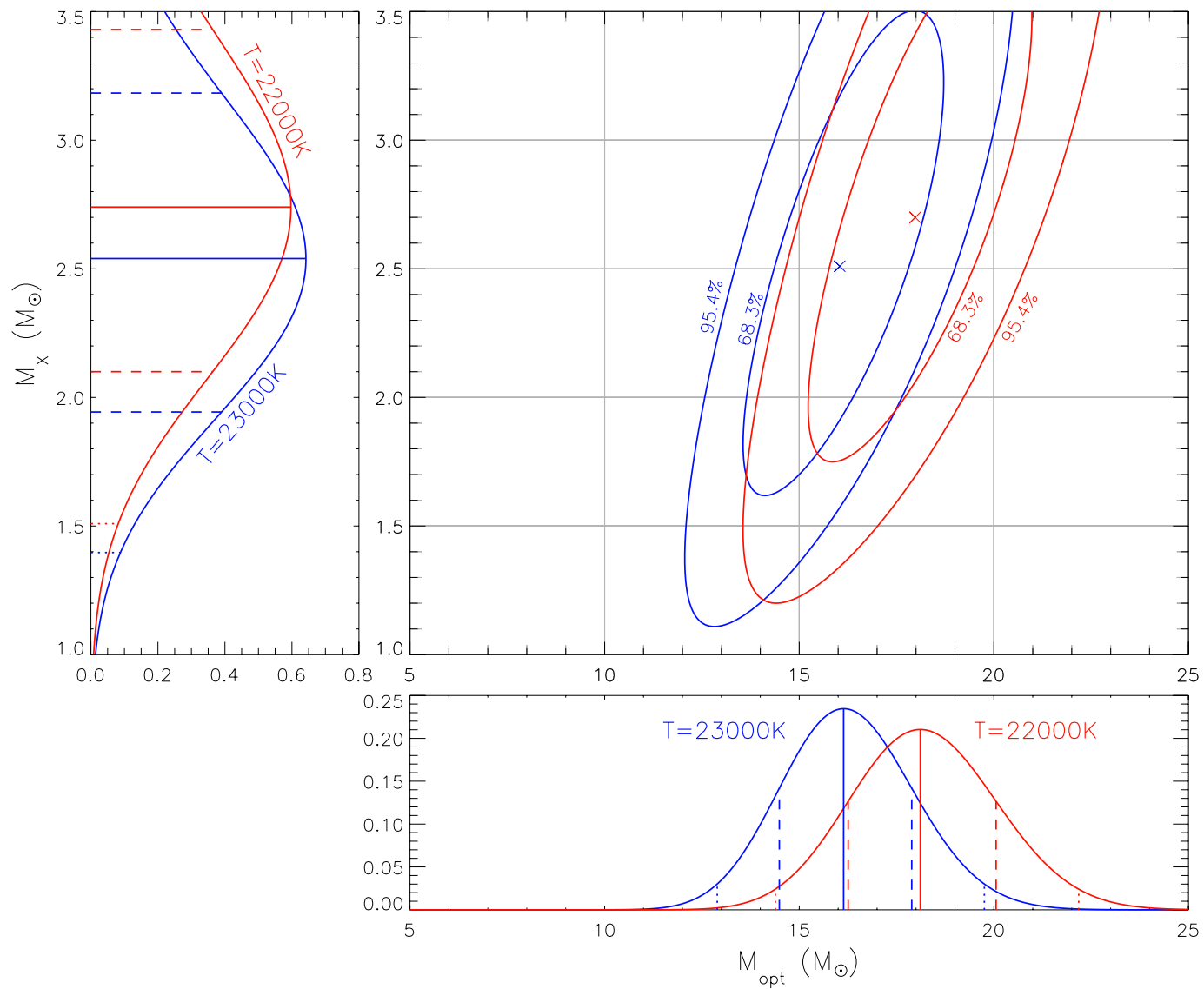

Figure 9. Probability density plot for neutron star mass $\left(M_{\mathrm{X}}\right)$ as a function of OB star mass $\left(M_{\mathrm{opt}}\right)$. Red contours show the $68.3 \%$ and $95.4 \%$ confidence intervals for masses using the best-fit template $(\log (g)=3.5$ and $T=22,000 \mathrm{~K})$. The panel on the left shows the PDF for $M_{\mathrm{X}}$ marginalized over $M_{\mathrm{opt}}$. Similarly, the lower panel shows the PDF for $M_{\mathrm{opt}}$ marginalized over $M_{\mathrm{X}}$. In these panels, the solid, dashed, and dotted lines show the peak and $68.3 \%$ and $95.4 \%$ confidence intervals, respectively. We obtain $M_{\mathrm{X}}=2.7_{-0.6}^{+0.7} M_{\odot}$ and $M_{\mathrm{opt}}=18.1_{-1.9}^{+2.0} M_{\odot}$. Contours for the less likely scenario with $\log (g)=4.0$ and $T=23,000 \mathrm{~K}$ are shown in blue. In this case, $M_{\mathrm{X}}=2.5 \pm 0.6 M_{\odot}$ and $M_{\mathrm{opt}}=16.1_{-1.7}^{+1.8} M_{\odot}$.

(A color version of this figure is available in the online journal.) 
Table 3

System Parameters for SMC X-1 and LMC X-4

\begin{tabular}{lcc}
\hline \hline Property & SMC X-1 & LMC X-4 \\
\hline Period $(P)$ & 3.89 days & 1.41 days \\
$P_{\text {spin }}$ & $0.708 \mathrm{~s}$ & $13.5 \mathrm{~s}$ \\
$a_{X} \sin i$ (lt-s) & $53.4876 \pm 0.0004$ & $26.343 \pm 0.016$ \\
Eclipse half-angle $\left(\theta_{e}\right)$ & $26^{\circ}-30.5$ & $27^{\circ} \pm 2^{\circ}$ \\
Mean systemic velocity $\left(\gamma_{\text {opt }}\right)$ & $-191 \pm 6 \mathrm{~km} \mathrm{~s}^{-1}$ & $306 \pm 10 \mathrm{~km} \mathrm{~s}^{-1}$ \\
Velocity semi-amplitude $\left(k_{\text {opt }}\right)$ & $20.2 \pm 1.1 \mathrm{~km} \mathrm{~s}^{-1}$ & $35.1 \pm 1.5 \mathrm{~km} \mathrm{~s}^{-1}$ \\
\hline Companion spectral type & B0I & O8III \\
Companion $T_{\text {eff }}$ & $29000 \mathrm{~K}$ & $35000 \mathrm{~K}^{\mathrm{b}}$ \\
Distance $(d)$ & $60.6 \pm 1 \mathrm{kpc}^{\mathrm{a}}$ & $49.4 \pm 1 \mathrm{kpc}^{\mathrm{b}}$ \\
Visual magnitude $\left(m_{V}\right)^{\mathrm{c}}$ & $13.3 \pm 0.1$ & $14.0 \pm 0.1$ \\
Extinction $\left(A_{V}\right)^{\mathrm{d}}$ & $0.12 \pm 0.01$ & $0.25 \pm 0.04$ \\
\hline NS mass $\left(M_{\mathrm{X}}\right)$ & & \\
van der Meer et al. $(2007)$ & $1.06_{-0.10}^{+0.11} M_{\odot}$ & $1.25_{-0.10}^{+0.11} M_{\odot}$ \\
Our calculation & $1.32_{-0.14}^{+0.16} M_{\odot}$ & $2.05_{-0.23}^{+0.24} M_{\odot}$ \\
OB star mass $\left(M_{\text {opt }}\right)$ & $\approx 15.7 M_{\odot}$ & $\approx 14.5 M_{\odot}$ \\
van der Meer et al. $(2007)$ & $21.1_{-2.9}^{+3.2} M_{\odot}$ & $29.1_{-4.4}^{+4.8} M_{\odot}$ \\
Our calculation & & \\
\hline
\end{tabular}

Notes. Data from van der Meer et al. (2007).

${ }^{\text {a }}$ Hilditch et al. (2005).

${ }^{\mathrm{b}}$ Freedman et al. (2001).

c Conservative 0.1 mag errors assumed.

${ }^{\mathrm{d}}$ Foreground extinction to galaxy only. Error bars are approximate, the dominant uncertainty has been assigned to $m_{V}$.

volume radius will be smaller than the effective photometric radius. Using a larger radius for the Roche lobe increases the masses of both the components in the binary.

Putting it another way, if we use the Roche volume radius to calculate the brightness of the star, we will get a number lower than the observed brightness. A similar discrepancy is observed by Massey et al. (2012) in massive binaries in the LMC. They find that the absolute magnitude of LMC 172231 calculated using a spherical approximation is 0.45 mag fainter than observed, while for the triple system [ST92]2-28, the numbers are consistent within errors. Using system parameters derived by van der Meer et al. (2007), we find a similar offset of $0.45 \mathrm{mag}$ for LMC X-4 and $0.2 \mathrm{mag}$ for SMC X-1. If we incorporate this uncertainty by allowing offsets of $0-0.4$ mag, we get $M_{\mathrm{X}}=2.2_{-0.6}^{+0.8} M_{\odot}$ and $M_{\mathrm{opt}}=13 \pm 4 M_{\odot}$.

Thus while this method has potential, more detailed modeling of the primary is clearly required to accurately infer component masses.

\section{CONCLUSION}

From our spectroscopic measurements we find that the donor star in XMMU J013236.7+303228 is a B1.5IV subgiant with effective temperature $T=22,000-23,000 \mathrm{~K}$. Assuming a circular orbit, we measure a mean systemic velocity $\gamma_{\mathrm{opt}}=$ $-80 \pm 5 \mathrm{~km} \mathrm{~s}^{-1}$ and velocity semi-amplitude $K_{\mathrm{opt}}=63 \pm$ $12 \mathrm{~km} \mathrm{~s}^{-1}$ for the B star. M 33 is nearly face-on, with recession velocity of $-179 \mathrm{~km} \mathrm{~s}^{-1}$ (de Vaucouleurs et al. 1991) — so this binary seems to be moving away from the disk at $100 \mathrm{~km} \mathrm{~s}^{-1}$.

Using the physical properties of the B star determined from our optical spectroscopy we find a mass for the donor of $M_{\text {opt }}=11 \pm 1 M_{\odot}$. This mass is based on stellar evolution models and will be reasonably accurate so long as binary evolution has not significantly altered the mass-luminosity relation. However, it is difficult to test this assumption based on any available observations. Using this spectroscopic mass, we calculate the mass of the compact object, $M_{\mathrm{X}}=2.0 \pm 0.4 M_{\odot}$. This is higher than the canonical $1.4 M_{\odot}$ for neutron stars, but comparable to masses of other neutron stars in X-ray binaries such as the HMXB Vela X-1 $\left(1.88 \pm 0.13 M_{\odot}\right.$; Barziv et al. 2001; Quaintrell et al. 2003) or the low-mass X-ray binaries Cyg X-2 (1.71 $\pm 0.21 M_{\odot}$; Casares et al. 2010) and 4U 1822-371 $\left(1.96 \pm 0.35 M_{\odot} ;\right.$ Muñoz-Darias et al. 2005). Since no pulsations have been detected, we have only indirect evidence, based on the X-ray spectrum, that the compact object is a neutron star. However, the mass we derive here is smaller than would be expected for a black hole.

Based on the stable X-ray flux, we infer that the donor is transferring mass to the neutron star by Roche lobe overflow. By equating the Roche lobe radius to physical radius of $R=9.1 \pm 0.3 R_{\odot}$, derived from the known distance to M 33, combined with the stellar luminosity and temperature, we derive an additional orbital constraint. From a first-pass calculation with a spherical approximation for the shape of the primary, we find substantially larger masses. However, applying this technique to the well-studied binaries LMC X-4 and SMC X1 , both of which have measured component masses, we find that it consistently overestimates the compact object mass. This is likely because the Roche surface is not spherical but elongated, which is not taken into account in our estimate. Attempting to account for that, we infer $M_{\mathrm{X}}=2.7_{-0.6}^{+0.7} M_{\odot}$ and $M_{\text {opt }}=18.1 \pm 2.0 M_{\odot}$. Future efforts to more accurately model the system geometry will improve the accuracy of this technique, which is applicable generally to Roche lobe overflow systems with known distances.

We thank Brian Grefenstette for helping us to interpret the $X M M$ data.

Some of the data presented herein were obtained at the W. M. Keck Observatory, which is operated as a scientific partnership among the California Institute of Technology, the University of California, and the National Aeronautics and Space Administration. The Observatory was made possible by the generous financial support of the W. M. Keck Foundation.

This research has made use of the NASA/IPAC Extragalactic Database (NED) which is operated by the Jet Propulsion Laboratory, California Institute of Technology, under contract with the National Aeronautics and Space Administration. This research has made use of NASA's Astrophysics Data System Bibliographic Services. This research used the facilities of the Canadian Astronomy Data Centre operated by the National Research Council of Canada with the support of the Canadian Space Agency.

\section{REFERENCES}

Barziv, O., Kaper, L., Van Kerkwijk, M. H., Telting, J. H., \& Van Paradijs, J. 2001, A\&A, 377, 925

Bessell, M. S., Castelli, F., \& Plez, B. 1998, A\&A, 250, 231

Brott, I., de Mink, S. E., Cantiello, M., et al. 2011, A\&A, 530, A115

Casares, J., Hernández, J. I. G., Israelian, G., \& Rebolo, R. 2010, MNRAS, 401, 2517

Cox, A. N. 2000, Allen's Astrophysical Quantities (4th ed.; Berlin: Springer)

Demorest, P. B., Pennucci, T., Ransom, S. M., Roberts, M. S. E., \& Hessels, J. W. T. 2010, Nature, 467, 1081

de Vaucouleurs, G., de Vaucouleurs, A., Corwin, H. G. J., et al. 1991, Third Reference Catalogue of Bright Galaxies (New York, NY: Springer), Vols. I-III

Eggleton, P. P. 1983, ApJ, 268, 368

Figueira, P., Pepe, F., Lovis, C., \& Mayor, M. 2010, A\&A, 515, A106 Freedman, W. L., Madore, B. F., Gibson, B. K., et al. 2001, ApJ, 553, 47 
Freire, P. C. C., Ransom, S. M., Bégin, S., et al. 2008, ApJ, 675, 670

Hilditch, R. W., Howarth, I. D., \& Harries, T. J. 2005, MNRAS, 357, 304

Jester, S., Schneider, D. P., Richards, G. T., et al. 2005, AJ, 130, 873

Joss, P. C., \& Rappaport, S. A. 1984, ARA\&A, 22, 537

Landsman, W. B. 1993, in ASP Conf. Ser. 52, Astronomical Data Analysis Software and Systems II, ed. R. J. Hanisch, R. J. V. Bissenden, \& J. Barnes (San Francisco, CA: ASP), 246

Lang, D., Hogg, D. W., Mierle, K., Blanton, M., \& Roweis, S. 2010, AJ, 139,1782

Lattimer, J., \& Prakash, M. 2005, Phys. Rev. Lett., 94, 111101

Lattimer, J. M., Prakash, M., Day, R. R., \& Year, M. 2010, arXiv:1012.3208

Markwardt, C. B. 2009, in ASP Conf. Ser. 411, Astronomical Data Analysis Software and Systems XVIII, ed. D. A. Bohlender, D. Durand, \& P. Dowler (San Francisco, CA: ASP), 251

Mason, A. B., Norton, A. J., Clark, J. S., Negueruela, I., \& Roche, P. 2011, A\&A, 532, A 124

Massey, P., Morrell, N. I., Neugent, K. F., et al. 2012, ApJ, 748, 96

McCarthy, J. K., Cohen, J. G., Butcher, B., et al. 1998, Proc. SPIE, 3355, 81

McConnachie, A. W., Irwin, M. J., Ferguson, A. M. N., et al. 2005, MNRAS, 356,979
Munari, U., Sordo, R., Castelli, F., \& Zwitter, T. 2005, A\&A, 442, 1127

Muñoz-Darias, T., Casares, J., \& Martínez-Pais, I. G. 2005, ApJ, 635, 502

Oke, J. B., Cohen, J. G., Carr, M., et al. 1995, PASP, 107, 375

Pietsch, W., Misanovic, Z., Haberl, F., et al. 2004, A\&A, 426, 11

Pietsch, W., Haberl, F., Gaetz, T. J., et al. 2009, ApJ, 694, 449

Pietsch, W., Plucinsky, P. P., Haberl, F., Shporer, A., \& Mazeh, T. 2006, ATel, 905, 1

Quaintrell, H., Norton, A. J., Ash, T. D. C., et al. 2003, A\&A, 401, 313

Rockosi, C., Stover, R., Kibrick, R., et al. 2010, Proc. SPIE, 7735, 77350R

Shporer, A., Hartman, J., Mazeh, T., \& Pietsch, W. 2006, ATel, 913, 1

Steidel, C. C., Shapley, A. E., Pettini, M., et al. 2004, ApJ, 604, 534

Timmes, F. X., Woosley, S. E., \& Weaver, T. A. 1996, ApJ, 457, 834

Torres, G. 2010, AJ, 140, 1158

van der Meer, A., Kaper, L., van Kerkwijk, M. H., Heemskerk, M. H. M., \& van den Heuvel, E. P. J. 2007, A\&A, 473, 523

van Dokkum, P. G. 2001, PASP, 113, 1420

van Kerkwijk, M. H., Breton, R. P., \& Kulkarni, S. R. 2011, ApJ, 728, 95

Walborn, N. R., \& Fitzpatrick, E. L. 1990, PASP, 102, 379 